\title{
ON THE EXISTENCE OF OPTIMAL PROCESSES
}

\author{
ROLF KLÖTZLER
}

Sektion Mathematik, Karl-Marx-Universität, 701 Leipzig, DDR

Many proofs of optimal solutions for a real functional $J(x)$ on a set $X$ are based on the generalized theorem of Weierstrass. It states that, with a given topology of $X$, Min $J(x)$ exists if $J(x)$ is a lower semicontinuous functional and $X$ is a closed and $x \in X$

compact set. What can we do-especially in the theory of optimal control problems'-if some assumptions of this theorem are not fulfilled? One method is, roughly speaking, the following: with respect to the given minimization problem we construct another "similar problem", prove the existence of optimal solutions of that, and hence conclude, if possible, the existence of solutions of the first problem. For the construction of "similar problems" it is very useful to have the concept of variationa pairs and its extension in the sense of A. D. Joffe and W. M. Tichomirov [8], published 1968. These authors characterize, for a given Hausdorff space $X$ and a real functional $J(x)$ defined on $X$, the computation of $\inf J(x)$ by the so-called variational pair $(X, J)$. A variational pair $\left(Y, J^{*}\right)$ is called an extension of $(X, J)$ if there exists a continuous map $i: X \rightarrow Y$ satisfying the following conditions:

(i) $i(X)$ is dense in $Y$,

(ii) $J(x) \geqslant J^{*}(i(x)) \forall x \in X$.

(iii) For every neighbourhood $V$ of each point $y \in Y$ we have

$$
\inf _{l=1} J(x) \leqslant J^{*}(y) .\left({ }^{1}\right)
$$

These properties imply immediately $\inf _{x \in X} J(x)=\inf _{y \in X} J^{*}(y)$. Moreover, we easily obtain the fact that $y_{0}=i\left(x_{0}\right)$ is a (local) minimal point of the variational pair $\left(Y, J^{*}\right)$ if $x_{0}$ is a (local) minimal point of the variational pair $(X, J)$. On the other hand, we do not generally know the existence of $\underset{x \in X}{\operatorname{Min}} J(x)$ if $\underset{y \in Y}{\operatorname{Min}} J^{*}(y)$ exists. However, it is often possible to get sufficient conditions for the existence of $\underset{x \in X}{\operatorname{Min}} J(x)$ by starting from the existence of $\underset{y \in Y}{\operatorname{Min}} J^{*}(y)$. For example, this method is usual for finding strong

(1) $i^{-1}(V)$ denotes the set of all $x \in X$ with $i(x) \subset V$. 
solutions of variational problems in Sobolev spaces through the knowledge of weak solutions.

Special.constructions of extensions at $(X, J)$ have already been given for variational problems with simple integrals by N. N. Bogoljubov 1930, L. C. Young 1937 and E. J. McShane 1940 (see the references of [8]). In the last few years these ideas have been rediscovered and further developed for more general problems of optimization in the sense of optimal control problems. Important papers on them have been published not only by Joffe and Tichomirov [8] but also by R. W. Gamkrelidze 1962, J. Warga 1962, W. F. Krotov 1960, A. D. Joffe [7], J. Ekeland [6], H. Berliocchi and J. M. Lasry [3], and by V. Amdursky [1]. $\left({ }^{2}\right)$

Here and henceforward we use the construction of extensions in the sense of Bogoljubov, Joffe and Tichomirov. We study the optimal control problem

$$
J(x, u)=\int_{G} f(t, x, u) d t \rightarrow \text { Min }
$$

among all state functions $x(t)=\left(x^{1}, \ldots, x^{n}\right) \in W_{\nu}^{1}(G)$ and control functions $u(t)$ $=\left(u^{1}, \ldots, u^{l}\right) \in L_{y}(G)(v>m)$, where the vector-valued variable $t=\left(t^{1}, \ldots, t^{m}\right) \in G$ and $G$ is a bounded Lipschitz domain of $R^{m}$. Besides, $x(t)$ shall satisfy the side conditions $x_{t}=g(t, x, u)$ a.e. on $G$, the boundary condition $\left.x\right|_{\partial G}=\varphi(t)$ and the inclusion

$$
u(t) \in U=\left\{u(t) \in L_{\nu}(G) \mid u(t) \in V \subset R^{l}\right\} .
$$

We assume that $f, g \in C\left(G \times R^{n} \times R^{l}\right), \varphi(t) \in C^{1}(\partial G)$ and that $V$ is a closed nonvoid compact subset of $R^{l}$. We denote by $\mathfrak{A}_{0}$ the set of all admissible pairs $\langle x(t), u(t)\rangle$ ("processes" of (1)).

Then we construct for fixed $t$ and $x$ the set $B(t, x)=\bigcup_{v \in V} g(t, x, v)$. Since $g$ characterizes a continuous map $\left\langle G \times R^{n} \times R^{l}\right\rangle \rightarrow R^{*}$ with $x=m \cdot n$, it is evident that $B(t, x)$ is a closed and compact subset of $R^{*}$ for fixed $t$ and $x$. Moreover, we compute the new integrand

$$
f_{1}(t, x, \xi)=\operatorname{Min}_{\substack{v \in V \\ \xi=g(t, x, v)}} f(t, x, v) \quad \forall \xi \in B(t, x) .
$$

Since $V_{0}(t, x, \xi)=\{v \in V \mid \xi=g(t, x, v)\}$ is a closed and compact subset of $R^{x}$ and $f$ is continuous, $f_{1}(t, x, \xi)$ exists . everywhere. It is easy to see that $f_{1}(t, x, \xi)$ is lower semicontinuous with respect to the whole argument $\langle t, x, \xi\rangle$.

Then problem (1) is equivalent $\left({ }^{3}\right)$ to the problem

$$
J_{1}(x)=\int_{G} f_{1}\left(t, x, x_{t}\right) d t \rightarrow \text { Min on } \mathfrak{U}_{1}
$$

( ${ }^{2}$ See also the relevant lectures by J. M. Lasry at the Banach Center in Warsaw 1973, and by H. Berliocchi and by G. S. Goodman at this conference.

$\left(^{3}\right)$ This means that: If $\left\langle x_{0}, u_{0}\right\rangle$ is an optimal process of (1), then $x_{0}$ is an optimal solution of (2). Conversely, if $x_{0}$ is an optimal solution of (2), there exists a corresponding control function $u_{0}$, so that $\left\langle x_{0}, u_{0}\right\rangle$ is an optimal process of (1). with

$\mathfrak{A}_{1}=\left\{x(t)=\left(x^{1}, \ldots, x^{n}\right) \in W_{\nu}^{1}(G) \mid x_{t}(t) \in B(t, x(t))\right.$ on $\left.G,\left.x\right|_{\partial G}=\varphi(t)\right\}$.

Problem (2) represented an ulterior example for an extension of the variational pair to problem (1) with the map $i(x, u)=x$ as a projection.

Furthermore, we introduce the closed domain $A(t, x)=\operatorname{co} B(t, x) \subset R^{x}$ and the function $f_{2}(t, x, \eta)$ by the optimal problem

with

$$
f_{2}(t, x, \eta)=\sup _{\Omega}\{a+(b, \eta)\} \quad \forall \eta \in A(t, x)
$$

$$
\Omega=\left\{\langle a, b\rangle \in R^{1} \times R^{x} \mid a+(b, \xi) \leqslant f_{1}(t, x, \xi) \forall \xi \in B(t, x)\right\} .
$$

The function $f_{2}$ is called the support-function of $f_{1}$. The equation $z=f_{2}(t, x, \eta)$ characterizes for fixed $\langle t, x\rangle$ the lower boundary of the convex hull of the epigraph

$$
\operatorname{epi}\left(f_{1}\right)=\left\{\langle\xi, z\rangle \in R^{r+1} \mid \xi \in B(t, x), z \geqslant f_{1}(t, x, \xi)\right\} .
$$

The function $f_{2}(t, x, \eta)$ is a convex function of $\eta \in A(t, x)$ for fixed $\langle t, x\rangle$ and a lower semicontinuous function of $t$ and $x$ (see e.g. [12], § 4). Joffe and Tichomirov have proved in their paper cited above that the variational problem

$$
J_{2}(x)=\int_{G} f_{2}\left(t, x, x_{t}\right) d t \rightarrow \text { Min on } \mathfrak{U}_{2}
$$

with

$$
\mathfrak{U}_{2}=\left\{x(t) \in W_{\nu}^{1}(G)|x|_{\partial G}=\varphi(t), x_{t} \in A(t, x(t))\right\}
$$

characterizes an extended variational pair of (1) resp. (2) if the following condition holds:

(4) For any admissible state function $x(t)$ of (1) and a sequence of vector-valued functions $\xi_{k}(t)=g\left(t, x(t), u_{k}(t)\right)$ with $u_{k}(t) \in U$ satisfying $\xi_{k} \rightarrow x_{t}$ in $L_{\nu}(G)$ there is a sequence of processes of $(1)\left\langle\tilde{x}_{k}, \tilde{u}_{k}\right\rangle$ with the property $\left\|\tilde{x}_{k}-x\right\|_{c(G)} \rightarrow 0$, $\left\|u_{k}-\tilde{u}_{k}\right\|_{L, \nu(G)} \rightarrow 0$.

Certainly, this functional-analytical condition (4) is very useful, but it does not guarantee the existence of the minimum of our primary problem (1). Besides, it is generally (especially for $m>1$ ) very difficult to prove and to establish this condition (4). Therefore, we shall try another way of securing the existence of $\underset{2_{2}}{\operatorname{Min}_{2}} J_{2}(x)$ and the validity of the equation $\operatorname{Min} J_{2}(x)=\operatorname{Min} J(x, u)$. To this end we shall use some generalizations of the investigation carried out by R. Klötzler [9] and A. Angelus [2] concerning irregular variational problems.

Hypothesis I. Problem (3) has a solution $x_{0}(t)$.

By modifying the general existence theorems of L. Cesari [5] and C. Olech [11] we can guarantee this hypothesis if the assumptions of the following two lemmas are fulfilled. 
LEMMA 1. There are constants $C, D \geqslant 0$ such that

$$
|g(t, x, v)| \leqslant C+D|x| \quad \forall v \in V .
$$

Then $\mathfrak{\mathfrak { A }}_{2}$ is a weak compact and closed subset of $W_{\nu}^{1}(G)$ for $\nu>m$.

LEMMA 2. We assume that $\mathfrak{U}_{2}=\varnothing$ and that for all $\eta \in A(t, x)$ there exists a supporting plane $z=(\alpha(t, x),[\xi-\eta])+f_{2}(t, x, \eta)$ of the epigraph epi $\left(f_{2}\right)$ $=\left\{\langle\xi, z\rangle \in R^{\alpha+1} \mid \xi \in A(t, x), z \geqslant f_{2}(t, x, \xi)\right\}$ having measurable coefficients $\alpha_{l}(t, x)$ as the components of the vector $\alpha(t, x)$. Besides we suppose that there is a function $M(t) \in L_{\mu}(G)\left(v^{-1}+\mu^{-1}=1\right)$ such that $|\alpha(t, x)| \leqslant M(t) \forall\langle t, x\rangle \in R^{n+m}$. Finally, $f_{2}$ should be uniformly lower semicontinuous in $x$. Then $J_{2}(x)$ is a weakly lower semicontinuous functional on $\mathfrak{N}_{2} \subset W_{\nu}^{1}(G)$

A proof of these lemmas will be given in a later issue. Obviously by using Weierstrass' existence theorem we immediately obtain from Lemmas 1 and 2 the validity of Hypothesis I.

Conclusions from Hypothesis I

We introduce the set $\mathfrak{M}(t, x)=\left\{\xi \in B(t, x) \mid f_{1}(t, x, \xi)=f_{2}(t, x, \xi)\right\}$ for fixed arbitrary $\langle t, x\rangle$.

The following conclusions are evident.

(1) If the Joffe-Tichomirov condition (4) and Hypothesis 1 are satisfied, then $\inf J(x, u)=\inf J_{1}(x)=\operatorname{Min} J_{2}(x)$.

(2) Under Hypothesis I each solution $x_{0}(t)$ of (3) with the special property $x_{0 t}(t)$ $\in \mathfrak{M}\left(t, x_{0}(t)\right)$ almost everywhere on $G$ is also a solution of problem (2), i.e.,

$$
\underset{\mathfrak{A l}_{2}}{\operatorname{Min}_{2}} J_{2}(x)=J_{2}\left(x_{0}\right)=J_{1}\left(x_{0}\right)=\underset{\operatorname{Min}_{1}}{\operatorname{Min}} J_{1}(x) \text {. }
$$

For the proof of the second conclusion we observe that by definition $f_{2}(t, x, \xi)$ $\leqslant f_{1}(t, x, \xi)$ on $B(t, x)$ and $f_{2}(t, x, \xi)=f_{1}(t, x, \xi)$ on $\mathfrak{M}(t, x)$. Therefore, for every $x(t) \in \mathfrak{U}_{1}$ we have $J_{1}(x) \geqslant J_{2}(x) \geqslant \underset{\mathfrak{A}_{2}}{\operatorname{Min}} J_{2}(x)=J_{2}\left(x_{0}\right)=J_{1}\left(x_{0}\right)$.

Now the idea of the proof of optimal solutions to problem (2) resp. (1) is based on the following method. We demonstrate under some restrictions that either $x_{0 r}(t)$ $\in \mathfrak{M}\left(t, x_{0}(t)\right)$ almost everywhere and thus Conclusion 2 holds or that in other cases there exists another minimal solution $\tilde{x}_{0}$ of (3) fulfilling the assumption of Conclusion 2. Then $\tilde{x}_{0}$ is also a solution of problem (2) and thus also an optimal state function of (1).

Here we sketch this method only for the case $n=1$. $\left({ }^{4}\right)$

HYPOTHESIS II. For every $\eta \in A(t, x) \backslash \mathfrak{M}(t, x)$ there exists a supporting plane of epi $\left(f_{2}\right)$ with tangent points $\left\langle\eta, f_{2}(t, x, \eta)\right\rangle$ and $\left\langle p_{i}(t, x, \eta), f_{2}\left(t, x, p_{i}(t, x, \eta)\right)\right\rangle$ $\in \operatorname{epi}\left(f_{1}\right) \quad(i=1, \ldots, m+1)$ having the properties: $p_{l}(t, x, \eta) \in C^{1}$ with respect to $\langle t, x\rangle$, dimco $\left\{p_{1}, \ldots, p_{m+1}\right\}=m, \eta$ is an interior point of $\operatorname{co}\left\{p_{1}, \ldots, p_{m+1}\right\} \subset R^{m}$.

$\left.{ }^{4}\right)$ An accordingly larger description will be given in a later paper.
This supporting plane will be representable by the equation $z=a_{i}(t, x, \eta) \xi^{i}+$ $+b(t, x, \eta)$ with $a_{i}, b \in C^{1}$ for fixed $\eta$. Besides, the integrability conditions $p_{i \alpha_{t} \beta}+p_{i \alpha_{x}} p_{i \beta}$ $=p_{i \beta_{i} \alpha}+p_{i \beta_{x}} p_{i \alpha}$ for all $\alpha, \beta=1, \ldots, m, i=1, \ldots, m+1$ (here not summing resp. $i$ ) are fulfilled. Finally for every fixed $\eta$ we have either

$$
a_{\alpha_{t} \alpha}-b_{x} \equiv 0 \text { (case I), }
$$

$$
a_{\alpha_{t} \alpha}-b_{x} \neq 0 \text { everywhere (case II). }
$$

THEOREM. Under the Hypothesis I and II problem (2) has a solution and thus there exists also an optimal process of problem (1).

The main aspects of its proof are the following. In consequence of Hypothesis I there is a solution $x_{0}(t)$ of problem (3). We may assume that $x_{0 t}(t) \in \mathfrak{M}\left(t, x_{0}(t)\right)$ not almost everywhere on $G$, because in the contrary case $x_{0}$ itself is a solution of problem (2) by Conclusion 2. We introduce the set $\mathfrak{N}=\left\{t \in G \mid x_{0 t}(t) \notin \mathfrak{M}\left(t, x_{0}(t)\right)\right\}$. Our assumption implies mes $\mathfrak{N} \neq 0$. Since $x_{0} \in W_{v}^{1}(G)$ and $\nu>m$, Sobolev's embedding theorems permit us the conclusion $x_{0} \in C(\bar{G})$. From this and the property $x_{0 t}(t) \in A\left(t, x_{0}(t)\right)$ we infer that $x_{0 t}$ is bounded on $G$. Therefore, by using the " $\mathrm{Di}$ richlet growth" theorem of C. B. Morrey [10] (theorem 3.5.2, which is valid also for $p>v$ and $\mu=1$ ), it is easy to see that $x_{0}(t)$ is even a local Lipschitz continuous function on $\mathrm{G}$. This implies again, after H. Rademacher (see [10], theorem 3.1.6), the existence of all directional derivatives of $x_{0}(t)$ for almost all points $t \in G$. We denote by $\mathfrak{N}_{0}$ the subset of all points of $\mathfrak{N}$ in which all directional derivatives of $x_{0}(t)$ exist. With the above result the property mes $\mathfrak{R}_{0}=$ mes $\mathfrak{N} \neq 0$ is obvious.

We first take a point $t_{0} \in \mathfrak{N}_{0}$ with the corresponding derivative $\eta=x_{0 t}\left(t_{0}\right)$ such that the case II holds in Hypothesis II. Then we solve for every $i=1, \ldots, m+1$ and sufficiently small $|h|$ with $h \in R^{\mathbf{1}}$ the following $m+1$ initial value problems:

$$
x_{t}=p_{i}(t, x, \eta), \quad x\left(t_{0}\right)=x_{0}\left(t_{0}\right)+h \quad(i=1, \ldots, m+1) .
$$

In consequence of the integrability conditions of hypothesis II these initial value problems are solvable (see e.g. [4] $\S 27$ ); we denote their unique solutions by $\sigma_{l}(t, h, \eta)$. With them we construct the two integralconoid functions

$$
\psi_{1}(t, h, \eta)=\operatorname{Min}_{i} \sigma_{t}(t, h, \eta), \quad \psi_{2}(t, h, \eta)=\operatorname{Max}_{i} \sigma_{i}(t, h, \eta)
$$

in a neighbourhood of $t_{0}$. Analogously to [9] we can prove that for sufficiently small $|h|$ and $h>0$ (resp. $h<0$ ) there exists a special neighbourhood $U\left(t_{0}, h\right)$ of $t_{0}$ with the following properties.

1. $\psi_{1}(t, h, \eta)-x_{0}(t)>0$ for $h>0$ in $U\left(t_{0}, h\right)$ resp.

$\psi_{2}(t, h, \eta)-x_{0}(t)<0$ for $h<0$ in $U\left(t_{0}, h\right)$,

2. $\psi_{1,2}(t, h, \eta)-x_{0}(t)=0 \forall t \in \partial U\left(t_{0}, h\right)$ as the boundary of $U\left(t_{0}, h\right)$ 
Furthermore, we can prove (see [9], p. 16) that for $a_{\alpha_{t} \alpha}(t, x, \eta)-b_{x}(t, x, \eta) \underset{(<)}{>} 0$ and $h_{(<)}$the function

$$
\tilde{x}_{0}(t)= \begin{cases}x_{0}(t) & \text { in } G \backslash U\left(t_{0}, h\right), \\ \psi_{1(2)}(t, h, \eta) & \text { in } U\left(t_{0}, h\right)\end{cases}
$$

is an element of $\mathfrak{A}_{2}$ satisfying the relation $J_{2}\left(\tilde{x}_{0}\right)<J_{2}\left(x_{0}\right)$. But this is a contradiction of the minimal property of $x_{0}$ with respect to problem (3). This means that for $x_{0}(t)$ there are no points $t_{0} \in \mathfrak{R}_{0}$ at which $\eta=x_{0 t}\left(t_{0}\right)$ realizes case II of Hypothesis Ir.

With the result obtained before we know under Hypothesis II that case $I$ is valid for every $t_{0}$ with the corresponding derivative $\eta=x_{0 t}\left(t_{0}\right)$. Then the constructed neighbourhoods $U\left(t_{0}, h\right)$, here considered for all $t_{0} \in \mathfrak{N}_{0}$, define a covering of $\mathfrak{N}_{0}$. By using Vitali's covering theorem we can choose a countable set of disjunct neighbourhoods $U_{j}=U\left(t_{j}, h_{j}\right)$ in the sense above, such that mes $\left[\mathfrak{N}_{0}-\cup U_{j}\right]=0$. If we replace $x_{0}(t)$ in $U_{j}$ by $\psi_{1}\left(t, h_{j}, \eta_{j}\right)$ with $\eta_{j}=x_{0 t}\left(t_{j}\right)$, then we obtain, analogously to [9], a new solution $\tilde{x}_{0}$ of problem (3) with $\tilde{x}_{0 t}(t) \in \mathfrak{M}\left(t, \tilde{x}_{0}(t)\right)$ almost everywhere on $\mathrm{G}$. From conclusion 2 it follows that the function $\tilde{x}_{0}$ is a solution of problem (2) as well, and thus the proof of our theorem is finished.

Remark. It is possible to give examples in which Hypothesis $\mathrm{I}$ is fulfilled but no solutions of (2) exist because Hypothesis II is not valid (see [9], p. 8)

\section{References}

[1] V. A m d u r s k y, The existence of solutions to classical variational problems without assuming convexity, Bull. Amer. Math. Soc. 78. 3 (1972).

[2] A. A n g e l u s, Beiträge zur Theorie direkter Methoden bei irregulären Variationsproblemen, Beiträge zur Analysis 7 (1974).

[3] H. B e rli o c chi, J. M. Las ry, Sur le contrôle optimal de systemes gouvernés par des équations aux derivées partielles, C.R. Acad. Sci. A 273 (1971), pp. 1222-1225.

[4] C. Ca rat ho od ory, Variationsrechnung und partielle Differentialgleichungen erster Ordnung I, B.G. Teubner Verlagsgesellschaft, Leipzig 1956.

[5] L. C e s a ri, Existence theorems for multidimensional problems of optimal control; Differential Equations and Dynamical Systems, Academic Press, New York, 1967, pp. 115-132.

[6] I. E kela nd, Sur le contróle optimal de systemes gouvernés par des équations elliptiques, J. Functional Analysis 9 (1972), pp. 1-62.

[7] A. D. J of f e, Transformation von Variationsaufgaben I, II, Izvest. Akad. Nauk SSSR, Techn. Kybernetika 3 (1967), pp. 51-60; 4 (1967), pp. 15-23 (in Russian).

[8] -, W. M. T i ch o m i r ov, Erweiterung von Variationsproblemen, Trud. Mosk. Mat. Obsch. 18 (1968), pp. 187-246 (in Russian).

[9] R. K 1 ö z z l e r, Direkte Methoden bei irregulären Variationsproblemen, Beiträge zur Analysis 5 (1973), pp. 7-17.

[10] C. B. Morrey, Multiple integrals in the calculus of variations, Springer-Verl., Berlin-Heidelberg-New York 1966

[11] $\mathrm{C} . \mathrm{O} \mathrm{lech}$, Existence theorems for optimal control problems involving multiple integrals, Journal of Differential Equations 6. 3 (1969), pp. 512-526.

[12] J. S to e r, Ch. W it z g a l l, Convexity and optimization in finite dimensions, Springer-Verl., Berlin-Heidelberg-New York 1970.

\section{APPROXIMATION METHODS FOR NONLINEAR \\ PROBLEMS WITH CONSTRAINTS IN FORM \\ OF VARIATIONAL INEQUALITIES}

\author{
REINHARD KLUGE
}

Zentralinstitut für Mathematik und Mechanik der AdW der DDR, Berlin, DDR

We consider here a number of nonlinear problems, which can all be written in the form of mixed variational inequalities. We assume also the constraints on these problems to be expressed in the form of mixed variational inequalities.

In other words, we consider mixed variational inequalities on sets of solutions of other variational inequalities.

In this paper we confine ourselves to the case where the operators and functionals figuring in the variational inequalities are monotone and convex, respectively. Generalizations into various directions are possible.

Part 1 contains an existence theorem for solutions of variational inequalities; we shall show that many problems can be considered as such inequalities. In Part 2 we give some qualitative statements concerning variational inequalities on solution sets of other variational inequalities. As an example an optimal control problem for a linear operator equation is mentioned. Part 3 concerns certain projection methods for the approximate solution of the general problem stated in Part 2. In Part 4 iterative projection methods for the same problem are considered. Finally, Part 5 contains some remarks on generalizations of the theory.

\section{Variational inequalities}

Let $B$ be a real reflexive Banach space with dual space $B^{*}$. By $(w, v)$ we denote the value of the functional $w \in B^{*}$ at the element $v \in B$. Let $C \subset B$ be a non-empty set, $S \in\left(B \rightarrow B^{*}\right)$ an operator, $z$ an element of $B^{*}$ and $h(x)$ a proper functional, i.e., $h \in\left(B \rightarrow R_{1}\right), h(x)>-\infty, h(x) \not \equiv+\infty$.

We consider the following problem. Find $u \in C$ satisfying

$$
(z-S u, u-v) \geqslant h(u)-h(v) \quad \text { for all } v \in C .
$$

Relation (1) is called a (mixed) variational inequality. Such an inequality was introduced first by Lescarret in 1965 for the case of a Hilbert space without constraints $(C=B=H)$. 